\title{
Imaging the dephasing of spin wave modes in a square thin film magnetic element
}

\author{
A. Barman, V. V. Kruglyak, R. J. Hicken, ${ }^{*}$ and J. M. Rowe \\ School of Physics, University of Exeter, Stocker Road, Exeter EX4 4QL, United Kingdom
}

A. Kundrotaite, J. Scott, and M. Rahman

Department of Physics and Astronomy, University of Glasgow, Glasgow G12 8QQ, United Kingdom

(Received 29 July 2003; revised manuscript received 8 March 2004; published 26 May 2004)

\begin{abstract}
We have used time-resolved scanning Kerr effect microscopy to study dephasing of spin wave modes in a square $\mathrm{Ni}_{81} \mathrm{Fe}_{19}$ element of $10 \mu \mathrm{m}$ width and $150 \mathrm{~nm}$ thickness. When a static magnetic field $\mathbf{H}$ was applied parallel to an edge of the square, demagnetized regions appeared at the edges orthogonal to the field. When $\mathbf{H}$ was applied along a diagonal, a demagnetized region appeared along the opposite diagonal. Time-resolved images of the out-of-plane magnetization component showed stripes that lie perpendicular to $\mathbf{H}$ and indicate the presence of spin wave modes with wave vector parallel to the static magnetization. The transient Kerr rotation was measured at different positions along an axis parallel to $\mathbf{H}$, and the power spectra revealed a number of different modes. Micromagnetic simulations reproduce both the observed images and the mode frequencies. This study allows us to understand an anisotropic damping observed at the center of the square element in terms of dephasing of the resonant mode spectrum.
\end{abstract}

DOI: 10.1103/PhysRevB.69.174426 PACS number(s): 75.30.Ds, 78.47.+p, 85.70.Kh, 76.50. $+\mathrm{g}$

\section{INTRODUCTION}

Time-resolved scanning Kerr effect microscopy (TRSKEM) may be used to explore the spatial and temporal character of ultrafast magnetic processes, while patterned thin films allow spin wave excitations to be studied within a confined geometry and are of interest for applications in magnetic data storage. Magnetization dynamics have been studied in such elements by various techniques. ${ }^{1-7}$ Substantial effort has been made to study magnetization reversal in continuous films and small elements ${ }^{8,9}$ but the coherence $e^{10,11}$ of precessional switching and the origin of the damping ${ }^{12,13}$ are the subject of continuing debate. Time-resolved magnetic images ${ }^{14}$ show that precessional switching is incoherent even in a permalloy element that initially has uniform magnetization.

When a magnetic system is excited by a short magnetic field pulse, the evolution of the magnetization is determined by various material parameters as well as the shape and size of the system, which control the interplay between exchange and dipolar interactions. The small amplitude precessional motion provides quantitative information about magnetic parameters of physical and technological relevance, and can be used to study the excitation of nonuniform eigenmodes in patterned thin films of nonellipsoidal shape. These eigenmodes have been studied experimentally by Brillouin light scattering, ${ }^{15}$ TRSKEM ${ }^{3}$ ferromagnetic resonance, ${ }^{16,17}$ and inductive techniques. ${ }^{13,18}$ However to fully understand their character it is necessary to determine both their frequency and their spatial character from dynamic images. Theoretical understanding of the eigenmodes is still being developed. ${ }^{19-24}$ For a rectangular magnetic stripe, the spins at the edge of the stripe are often assumed to be either free or fully pinned. ${ }^{25}$ Theoretical work has been performed on stripes of both infinitesimal ${ }^{23}$ and finite ${ }^{24}$ thickness. In the latter case, the dynamic magnetization at the edges was described with effective boundary conditions of purely dipolar nature and good agreement was found with experimental results. ${ }^{26-28}$

Configurational anisotropy in nanometer and micrometer sized elements has been known for some time. ${ }^{29-31,7} \mathrm{Re}$ cently we observed that, in a square element of $10 \mu \mathrm{m}$ length and $150 \mathrm{~nm}$ thickness, the precessional damping also shows a fourfold anisotropy when the static field is applied in different directions within the sample plane. ${ }^{7}$ Time-resolved magnetic images showed the formation of stripes that suggested the presence of nonuniform eigenmodes. Here we characterize the eigenmodes by TRSKEM and micromagnetic simulations. For an element of $10 \mu \mathrm{m}$ width, and with an optical resolution of better than $1 \mu \mathrm{m}$, one may observe spin waves with wave vector $(k)$ in the range $6 \times 10^{3}-6$ $\times 10^{4} \mathrm{~cm}^{-1}$. The frequencies of these magnetostatic waves depend upon the product of $k$ and the film thickness. For a continuous film of $100 \mathrm{~nm}$ thickness and a static field of about $1 \mathrm{kOe}$, the frequencies of the backward volume magnetostatic (BWVMS) waves ${ }^{22}$ lie about $1 \mathrm{GHz}$ below that of the uniform mode. The dimensions of the present sample are sufficiently large that the exchange interaction has little effect upon these modes, and so we may study the influence of the dipolar interaction in isolation. This provides a valuable starting point for studies of much smaller elements of similar aspect ratio where dipolar and exchange effects must be considered simultaneously.

\section{EXPERIMENT}

An array of square glass $/ \mathrm{Ni}_{81} \mathrm{Fe}_{19}(150$ $\mathrm{nm}) / \mathrm{Al}_{2} \mathrm{O}_{3}(20 \mathrm{~nm})$ elements of $10 \mu \mathrm{m}$ length and edge-toedge separation of $60 \mu \mathrm{m}$ was prepared by electron beam lithography and sputtering. A magnetic field of 150 Oe was applied parallel to one edge of the elements during growth to set the orientation of the uniaxial anisotropy axis. A detailed description of the optical pump-probe technique and TRSKEM has been reported elsewhere ${ }^{7,32,33}$ and so here we 


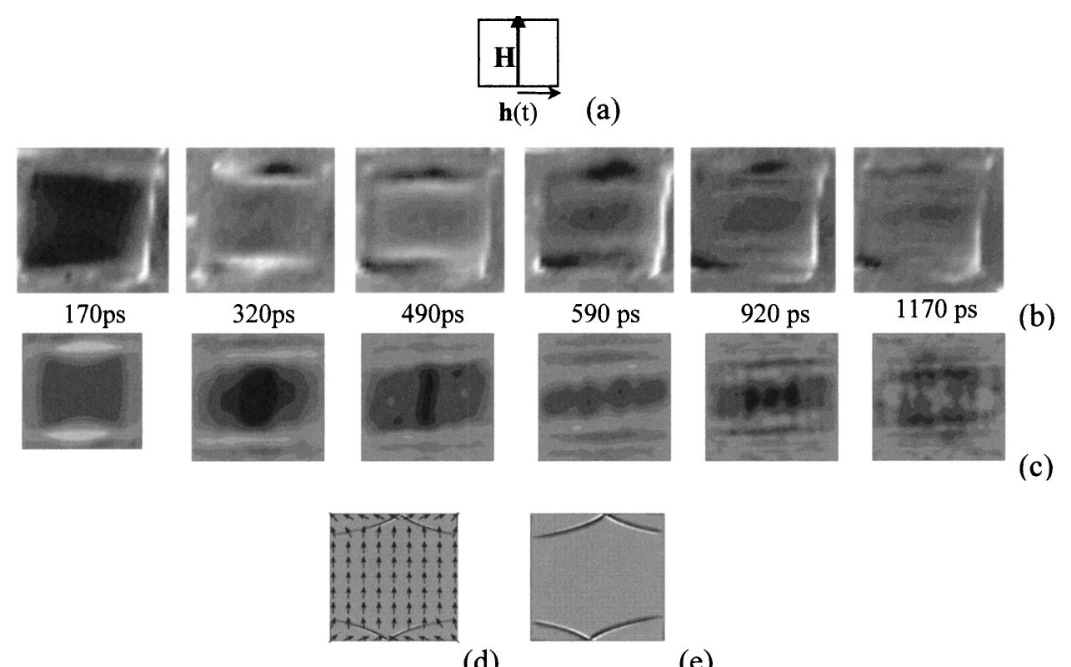

FIG. 1. (a) The experimental geometry $(H$ $=467 \mathrm{Oe}$ ), (b) the experimental dynamic images, and (c) the simulated dynamic images from OOMMF, at different time delays are shown. The static magnetization (d) and total internal field (e) in the static configuration are shown. The greyscale represents the out-of-plane component of the magnetization and internal field, respectively.

(d)

describe only the principal features of the experiment. The sample was excited by the pulsed magnetic field generated by the current from a GaAs photoconductive switch. The sample was overlaid on a coplanar strip transmission line and probed through its transparent substrate. The pulsed field profile was determined by an electro-optic sampling technique $^{33}$ and showed a rise time of about 40 ps and a decay time of about 2 ns. The peak magnetic field amplitude was estimated to be about 27 Oe. An in-plane static field $\mathbf{H}$ was applied to the sample and measurements were made as its strength $H$ and orientation were varied. The sample was scanned under a fixed sub- $\mu \mathrm{m}$ probe spot using a piezoelectric scanning stage to obtain dynamic magnetic images of the out-of-plane magnetization component.

\section{RESULTS AND DISCUSSIONS}

Measurements of the transient Kerr rotation at the center of the element were presented in Ref. 7. Fast Fourier transforms (FFT) of the transient response showed a single mode of precession. From the frequency of precession we deduced the presence of a fourfold anisotropy field of 33 Oe, with easy axes parallel to the edges of the element, in addition to a uniaxial anisotropy of about 2.5 Oe with easy axis parallel to the direction of the growth magnetic field. However dynamic images showed the magnetic response to be nonuniform. The experimental geometry is shown in Fig. 1(a) while dynamic images obtained at a static field value of $H$ $=467$ Oe are shown in Fig. 1(b). The greyscale represents the out-of-plane component of the magnetization. A stripe pattern rapidly becomes apparent within the images. The central stripe is broadest and the stripe width decreases toward the edge of the square. Since the stripes lie perpendicular to $\mathbf{H}$, the wave vectors of the excited modes must lie parallel to $\mathbf{H}$ in the BWVMS geometry.

The frequencies of the excited modes were determined by measuring the transient Kerr rotation with the probe spot placed 1,2,3, and $4 \mu \mathrm{m}$ from the center of the element on the symmetry axis parallel to $\mathbf{H}$. Figure 2(a) shows the pulsed field profile obtained by electro-optic sampling, while Figs. 2(b)-2(e) show the transient Kerr rotation. The right- hand panels show the power spectra obtained from FFTs using a Welch window function without zero padding. At $1 \mu \mathrm{m}$ from the center, the mode at $7.23 \mathrm{GHz}$ (mode 1), that was found at the center of the element, is still prominent, but at 2 $\mu \mathrm{m}$ from the center another peak at $6.36 \mathrm{GHz}$ (mode 2) has appeared. At $3 \mu \mathrm{m}$ from the center, mode 2 has grown in amplitude and become comparable to mode 1 while another peak at about 5.25 GHz (mode 3) also has significant amplitude. At $4 \mu \mathrm{m}$ from the center, mode 3 has become dominant. Another peak has appeared at about $3.8 \mathrm{GHz}$ but we did not observe this peak consistently at all values of $H$. The peaks below $3 \mathrm{GHz}$ are probably associated with secondary peaks in the pulsed field profile due to reflections of the current pulse from impedance mismatches on the transmission line. The positions of these peaks do not depend upon $H$, suggesting that they are not part of the resonant sample response. The frequencies of the additional modes shown in Fig. 2 are lower than that of the uniform mode, as expected for magnetostatic waves with wavelength in the $1-10 \mu \mathrm{m}$ range.

The dependence of the frequencies of modes 1-3 upon $H$
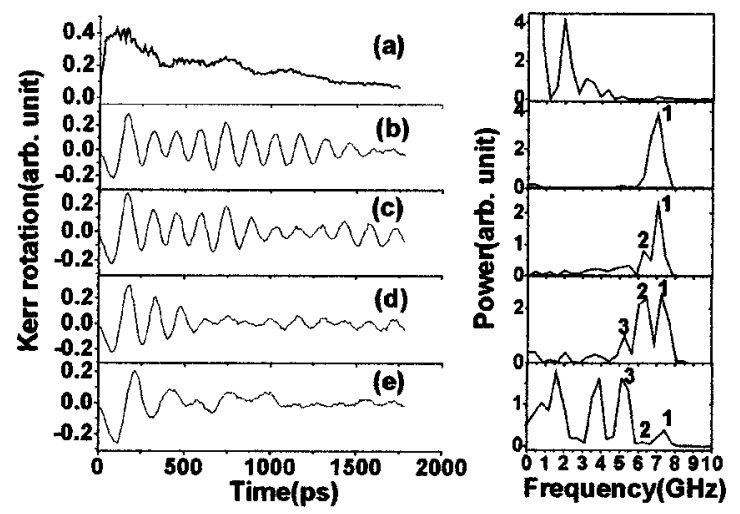

FIG. 2. The pulsed magnetic field profile (a) and time-dependent Kerr rotations are plotted in the left-hand panels for spots placed (b) $1 \mu \mathrm{m}$, (c) $2 \mu \mathrm{m}$, (d) $3 \mu \mathrm{m}$, and (e) $4 \mu \mathrm{m}$ from the center of the element on the symmetry axis parallel to $\mathbf{H}$. The orientation of $\mathbf{H}$ and $\mathbf{h}$ was as shown in Fig. 1(a). The right-hand panels show the corresponding FFT spectra. 


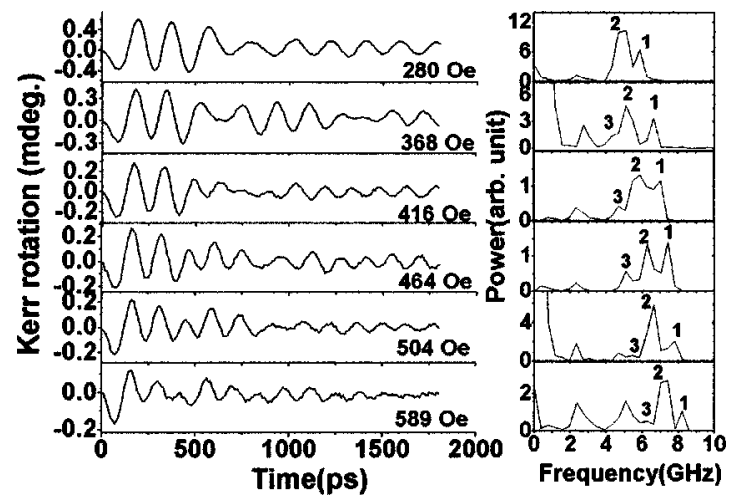

FIG. 3. The time-dependent Kerr rotations measured at $3 \mu \mathrm{m}$ from the center of the element on the symmetry axis parallel to $\mathbf{H}$ are shown in the left-hand panels. The orientation of $\mathbf{H}$ and $\mathbf{h}$ was as shown in Fig. 1(a). The right-hand panels show the corresponding FFT spectra.

was studied in measurements of the transient Kerr rotation at 3 and $4.5 \mu \mathrm{m}$ from the center of the element. Time-resolved images showed that the dynamic magnetization became highly irregular for $H$ values less than 100 Oe, presumably because the major part of the element was no longer saturated. Measurements were therefore made at field values of $H>100$ Oe. In Fig. 3 the transient Kerr rotation is plotted at different values of $H$ with the probe spot located $3 \mu \mathrm{m}$ from the center. The FFTs reveal the presence of modes 2 and 3 in addition to mode 1. In Fig. 4 the frequencies of modes 1-3 obtained from measurements at 3 and $4.5 \mu \mathrm{m}$ from the center, are represented by closed symbols. The frequencies obtained from the two measurement positions agree well.

To understand the variations in the frequencies and spatial character of the modes, we performed time-dependent micromagnetic simulations using the Object Oriented Micromagnetic Framework (OOMMF) software. ${ }^{34}$ The element was divided into a two-dimensional array of cells with dimensions of $40 \mathrm{~nm} \times 40 \mathrm{~nm} \times 150 \mathrm{~nm}$. Reduction of the cell size

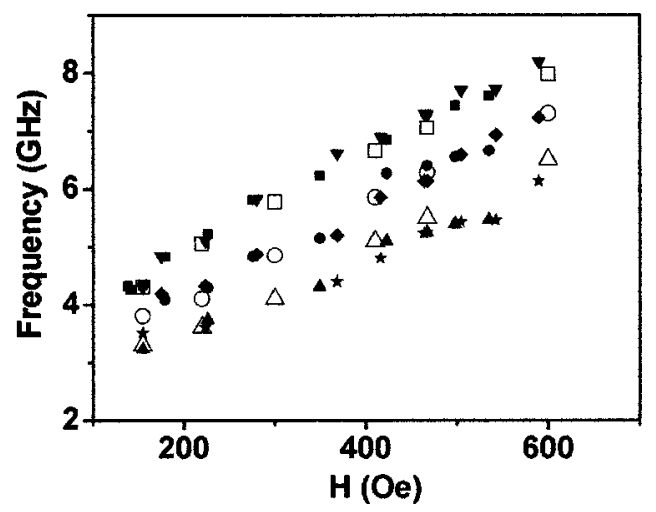

FIG. 4. Experimental frequencies obtained with probe spot 3 $\mu \mathrm{m}(\boldsymbol{\square}$, mode $1 ; \boldsymbol{\bullet}$, mode $2 ; \boldsymbol{\Lambda}$, mode 3$)$ and $4.5 \mu \mathrm{m}(\boldsymbol{\nabla}$, mode 1 ; $\downarrow$, mode $2 ; \star$, mode 3 ) from the center of the element, on the symmetry axis parallel to $\mathbf{H}$, are plotted as a function of $H$. Simulated frequencies from OOMMF at a location of $3 \mu \mathrm{m}$ from the center of the element are shown by the open symbols ( $\square$, mode 1 ; $\bigcirc$, mode $2 ; \triangle$, mode 3 ).



FIG. 5. OOMMF simulations of the time-dependent out-ofplane component of the normalized magnetization $\left(M_{z} / M_{S}\right)$ are shown. Results obtained at (a) $1 \mu \mathrm{m}$, (b) $2 \mu \mathrm{m}$, (c) $3 \mu \mathrm{m}$, and (d) 4 $\mu \mathrm{m}$ from the center of the element on the symmetry axis parallel to $\mathbf{H}(H=467 \mathrm{Oe})$ are shown in the left-hand panels. The right-hand panels show the corresponding FFT spectra.

and discretization through the thickness of the sample did not alter the results significantly. The finite thickness of the film affects the result through the demagnetizing field. The simulations assumed parameter values ${ }^{7}$ of $4 \pi M_{S}=10.8 \mathrm{kOe}, A$ $=1.3 \times 10^{-6} \mathrm{erg} / \mathrm{cm}, H_{K}=2.5 \mathrm{Oe}, g=2.15$, and a damping coefficient of 0.01 . The pulsed field $\mathbf{h}(t)$, had rise time of 40 ps and peak value of $27 \mathrm{Oe}$. The simulated images were spatially averaged with a Gaussian function with full width at half maximum of $700 \mathrm{~nm}$ corresponding to the nominal optical resolution of our TRSKEM. In Fig. 1(c) the simulated images are shown for time delays similar to those of the experimental images. The very good agreement between experimental and simulated images justifies the cell size and parameter values used in the simulation. In Figs. 1(d) and 1(e) we have shown the out-of-plane components of the static magnetization and the total internal field before application of the pulsed field. The simulated images in Fig. 1(c) clearly show that nonuniformity propagates from regions near the edges perpendicular to $\mathbf{H}$. The static magnetization and total internal field images show that these are demagnetized regions.

To reproduce the measured mode frequencies with the OOMMF simulation, the time-dependent magnetization was spatially averaged over a $1 \mu \mathrm{m}$ square mask of comparable area to that of the focused probe spot. The mask center was placed at distances of $1,2,3$, and $4 \mu \mathrm{m}$ from the center of the element. In Fig. 5 the time-dependent magnetization and corresponding power spectra are shown for the geometry in Fig. 1(a). The spectra show the appearance of modes 1-3 at frequencies close to those in Figs. 2(b)-2(e). The experimental and simulated frequencies at $H=467$ Oe are shown in Table I. OOMMF simulations were performed for six values of $H$ in the range of 155-600 Oe to reproduce the variation of mode frequency with $H$. The frequencies obtained from the power spectra at $3 \mu \mathrm{m}$ from the center are plotted as open symbols in Fig. 4. The simulated and measured frequencies are in reasonably good agreement. Mode 3 shows the greatest discrepancy, perhaps because it is more strongly localized at the edge of the element and more susceptible to perturbations of the dipolar field associated with edge defects. 
TABLE I. The mode frequencies obtained from experiment and simulation are shown in the case that a field of 467 Oe was applied parallel to an edge of the element.

\begin{tabular}{cccc}
\hline \hline & Mode 1 & Mode 2 & Mode 3 \\
\hline Experiment & 7.23 & 6.36 & 5.25 \\
OOMMF & 7.05 & 6.28 & 5.48 \\
\hline \hline
\end{tabular}

When $\mathbf{H}$ was instead applied parallel to a diagonal, a stronger apparent damping of the magnetization ${ }^{7}$ was observed at the center of the element and explained qualitatively in terms of the generation of nonuniform modes. In Fig. 6(a) we show the experimental geometry used in these measurements in which the static field strength was set to $H=155$ Oe. The experimental images at different time delays are shown in Fig. 6(b). The images show nonuniformity along the diagonal perpendicular to $\mathbf{H}$ even after 70 ps. Stripes appear first at the center of the element and the nonuniformity extends toward the corners with increasing time delay. Simulated images are shown in Fig. 6(c) and show similar features to the experiment. The out-of-plane components of the static magnetization and the total field are shown in Figs. 6(d) and 6(e), respectively. It is clear that nonuniformity again first appears at the edges of the demagnetized regions present in the static configuration.

To understand how nonuniformity develops we calculated the time-dependent magnetization within a $1 \mu \mathrm{m}$ area at the center of the element and at distances of $\sqrt{ } 2,2 \sqrt{ } 2,3 \sqrt{ } 2$, and $4 \sqrt{ } 2 \mu \mathrm{m}$ from the center of the element along the diagonal parallel to $\mathbf{H}$. The time dependence of the magnetization and the corresponding power spectra are shown in Figs. 7(b)7(f). The power spectra reveal a number of modes, the most prominent of which have center frequencies of 2.78, 3.13, 3.69, 4.22, and $4.92 \mathrm{GHz}$. A measurement made at the center of the element with $H=154$ Oe showed a clear peak at about $3.5 \mathrm{GHz}$ and a shoulder at about $4.7 \mathrm{GHz}$, as shown in Fig. 7(a).
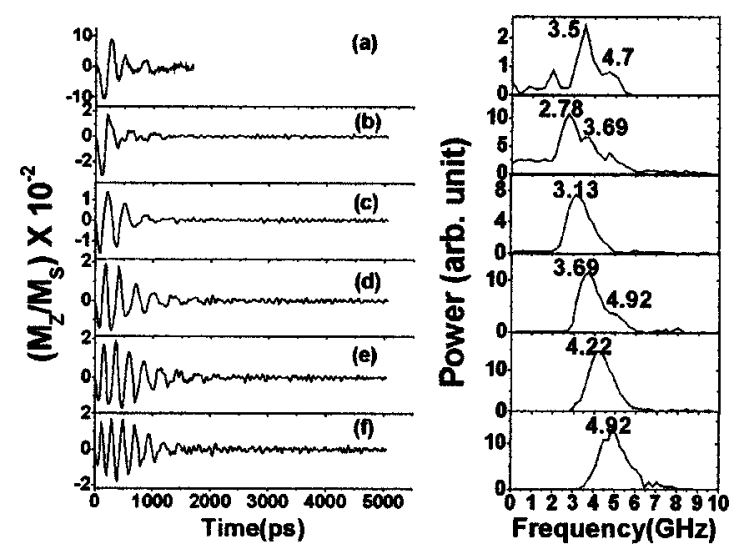

FIG. 7. Experimental polar Kerr rotation (a) at the center of the element and OOMMF simulations of the time-dependent normalized out-of-plane component of magnetization $\left(M_{z} / M_{S}\right)$ (b)-(f) are shown. Results obtained at (b) the center, (c) $\sqrt{2} \mu \mathrm{m}$, (d) $2 \sqrt{2} \mu \mathrm{m}$, (e) $3 \sqrt{ } 2 \mu \mathrm{m}$, and (f) $4 \sqrt{ } 2 \mu \mathrm{m}$ from the center of the element on the diagonal parallel to $\mathbf{H}(H=155 \mathrm{Oe})$ are shown in the left-hand panels. The right-hand panels show the corresponding FFT spectra. The peak frequencies have been marked.

Further simulations ${ }^{35}$ were performed with a harmonic driving field in order to excite individual modes and explore their spatial character. All modes were found to couple to the spatially uniform time-dependent magnetic field. Therefore when a pulsed field is applied, a number of modes are excited simultaneously and stripes appear in the experimental and simulated images as the modes dephase. With $\mathbf{H}$ applied parallel to an edge, only the highest frequency mode was found to have significant amplitude at the center of the element, whereas a number of modes had significant amplitude at the center when $\mathbf{H}$ was applied parallel to a diagonal. Dephasing hence leads to a larger apparent damping at the center of the element when $\mathbf{H}$ is applied parallel to a diagonal as compared to when $\mathbf{H}$ is applied parallel to an edge.

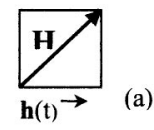

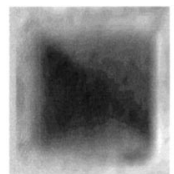

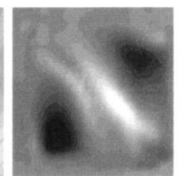

$70 \mathrm{ps}$

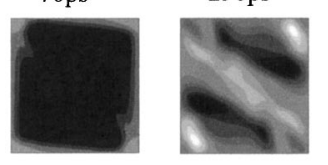

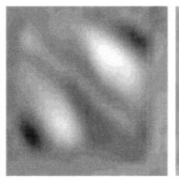

$450 \mathrm{ps}$


(d)

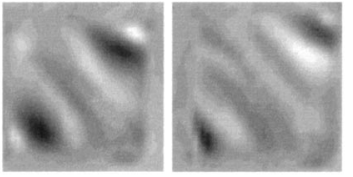

$$
510 \mathrm{ps}
$$

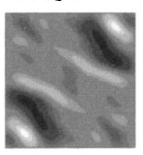

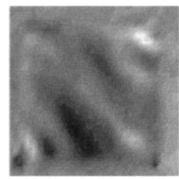

$960 \mathrm{ps} \quad(\mathrm{b})$



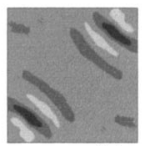

(c)
FIG. 6. The experimental dynamic images (b) and the simulated dynamic images (c) obtained in the experimental geometry (a) are shown. The static magnetization (d) and total internal field (e) in the static configuration are shown. The greyscale represents the out-of-plane components of the magnetization and internal field, respectively. 


\section{CONCLUSIONS}

We have investigated the nonuniform magnetic response of a $10 \mu \mathrm{m}$ square $\mathrm{Ni}_{81} \mathrm{Fe}_{19}$ element of $150 \mathrm{~nm}$ thickness to a pulsed magnetic field with 40 ps rise time. Time-resolved magnetic images showed the formation of stripes, lying normal to $\mathbf{H}$. With $\mathbf{H}$ applied parallel to the edge of the element, the transient Kerr rotation was recorded at four positions on the symmetry axis parallel to $\mathbf{H}$. The power spectra revealed the presence of two additional modes with frequency lower than that observed at the center of the element. Timedependent micromagnetic simulations reproduced the stripes observed in the experimental images and the frequencies of the resonant modes. When $\mathbf{H}$ was applied parallel to a diagonal of the element, the time-resolved magnetic images showed the formation of a denser stripe pattern, again lying normal to $\mathbf{H}$. Immediately after application of the pulsed field, the dynamic magnetization was found to be divided into two distinct regions lying on opposite sides of the diagonal perpendicular to $\mathbf{H}$. Simulations again reproduced the

*Email address: r.j.hicken@exeter.ac.uk

${ }^{1}$ W. K. Hiebert, A. Stankiewicz, and M. R. Freeman, Phys. Rev. Lett. 79, 1134 (1997).

${ }^{2}$ Y. Acremann, C. H. Back, M. Buess, O. Portmann, A. Vaterlaus, D. Pescia, and H. Melchior, Science 290, 492 (2000).

${ }^{3}$ J. P. Park, P. Eames, D. M. Engebretson, J. Berezovsky, and P. A. Crowell, Phys. Rev. Lett. 89, 277201 (2002).

${ }^{4}$ S. M. Cherif, Y. Roussigne, C. Dugautier, and P. Moch, J. Magn. Magn. Mater. 242-245, 591 (2002).

${ }^{5}$ M. M. Midzor, P. E. Wigen, D. Pelekhov, W. Chen, P. C. Hammel, and M. L. Roukes, J. Appl. Phys. 87, 6493 (2000).

${ }^{6}$ S. Jung, B. Watkins, L. DeLong, J. B. Ketterson, and V. Chandrasekhar, Phys. Rev. B 66, 132401 (2002).

${ }^{7}$ A. Barman, V. V. Kruglyak, R. J. Hicken, A. Kundrotaite, and M. Rahman, Appl. Phys. Lett. 82, 3065 (2003).

${ }^{8}$ W. K. Hiebert, G. E. Ballentine, and M. R. Freeman, Phys. Rev. B 65, 140404 (2002).

${ }^{9}$ Th. Gerrits, H. A. M. van den Berg, J. Hohlfeld, L. Bar, and Th. Rasing, Nature (London) 418, 509 (2002).

${ }^{10}$ T. J. Silva, P. Kabos, and M. R. Pufall, Appl. Phys. Lett. 81, 2205 (2002)

${ }^{11}$ W. K. Hiebert, G. E. Ballentine, L. Lagae, R. W. Hunt, and M. R. Freeman, J. Appl. Phys. 92, 392 (2002).

${ }^{12}$ J. Wu, N. D. Hughes, J. R. Moore, and R. J. Hicken, J. Magn. Magn. Mater. 241, 96 (2002).

${ }^{13}$ M. Covington, T. M. Crawford, and G. J. Parker, Phys. Rev. Lett. 89, 237202 (2002)

${ }^{14}$ W. K. Hiebert, L. Lagae, J. Das, J. Bekaert, R. Wirix-Speetjens, and J. De Boeck, J. Appl. Phys. 93, 6906 (2003).

${ }^{15}$ J. Jorzick, S. O. Demokritov, B. Hillebrands, M. Bailleul, C. Fermon, K. Y. Guslienko, A. N. Slavin, D. V. Berkov, and N. L. Gorn, Phys. Rev. Lett. 88, 047204 (2002).

${ }^{16}$ S. Tamaru, J. A. Bain, R. J. M. van de Veerdonk, T. M. Crawford, M. Covington, and M. H. Kryder, J. Appl. Phys. 91, 8034 (2002)

${ }^{17}$ Y. Zhai, J. Shi, X. Y. Zhang, L. Shi, Y. X. Xu, H. B. Huang, Z. H. stripes observed in the experimental images while simulations of the time-dependent magnetization, at five different positions along the direction parallel to $\mathbf{H}$, revealed the presence of five different resonant modes. The stripes observed in the images result from the excitation of a superposition of resonant modes by the pulsed field and their subsequent dephasing. The resonant modes have very different spatial character in the two geometries which leads to the observation of an anisotropy in the apparent damping at the center of the element. Consequently dephasing of the resonant mode spectrum must always be considered when measurements are made to determine the damping present in finite magnetic elements.

\section{ACKNOWLEDGMENTS}

We acknowledge the support of the Engineering and Physical Sciences Research Council and the supply of modified versions of the OOMMF code by Dr. M. Donahue.
Lu, and H. R. Zhai, J. Phys.: Condens. Matter 14, 7865 (2002).

${ }^{18}$ T. M. Crawford, M. Covington, and G. J. Parker, Phys. Rev. B 67, 024411 (2003).

${ }^{19}$ L. R. Walker, Phys. Rev. 105, 390 (1957).

${ }^{20}$ C. Kittel, Phys. Rev. 110, 1295 (1958).

${ }^{21}$ R. W. Damon and J. R. Eshbach, J. Phys. Chem. Solids 19, 308 (1961).

${ }^{22}$ B. A. Kalinikos and A. N. Slavin, J. Phys. C 19, 7013 (1986).

${ }^{23}$ P. H. Bryant, J. F. Smyth, S. Schultz, and D. R. Fredkin, Phys. Rev. B 47, 11255 (1993).

${ }^{24}$ K. Y. Guslienko, S. O. Demokritov, B. Hillebrands, and A. N. Slavin, Phys. Rev. B 66, 132402 (2002).

${ }^{25}$ A. G. Gurevich and G. A. Melkov, Magnetization Oscillations and Waves (CRC Press, New York, 1996).

${ }^{26}$ C. Mathieu, J. Jorzick, A. Frank, S. O. Demokritov, A. N. Slavin, B. Hillebrands, B. Bartenlian, C. Chappert, D. Decanini, F. Rousseaux, and E. Cambril, Phys. Rev. Lett. 81, 3968 (1998).

${ }^{27}$ J. Jorzick, S. O. Demokritov, C. Mathieu, B. Hillebrands, B. Bartenlian, C. Chappert, F. Rousseaux, and A. N. Slavin, Phys. Rev. B 60, 15194 (1999).

${ }^{28}$ Y. Roussigné, S. M. Chérif, C. Dugatier, and P. Moch, Phys. Rev. B 63, 134429 (2001).

${ }^{29}$ M. E. Schabes and H. N. Bertram, J. Appl. Phys. 64, 1347 (1988).

${ }^{30}$ R. P. Cowburn, A. O. Adeyeye, and M. E. Welland, Phys. Rev. Lett. 81, 5414 (1998).

${ }^{31}$ X. Zhu, P. Grütter, V. Metlushko, and B. Ilic, Appl. Phys. Lett. 80, 4789 (2002).

${ }^{32}$ R. J. Hicken and J. Wu, J. Appl. Phys. 85, 4580 (1999).

${ }^{33}$ A. Barman, V. V. Kruglyak, R. J. Hicken, A. Kundrotaite, and M. Rahman, IEE Proc.: Sci., Meas. Technol. 150, 260 (2003).

${ }^{34}$ M. Donahue and D. G. Porter, OOMMF User's guide, Version 1.0, Interagency Report NISTIR 6376, National Institute of Standards and Technology, Gaithersburg, MD, 1999: URL: http://math.nist.gov/oommf.

${ }^{35}$ A. Barman and R. J. Hicken (unpublished). 